\title{
Relapsed Mantle Cell Lymphoma, Allogenic Stem Cell Transplantation and COVID-19
}

\author{
James Dillon $^{1}$, Elizabeth Higgins ${ }^{1}$, Carmel-Ann Galligan ${ }^{1}$, Majella Moran ${ }^{1}$, Brendan Crowley ${ }^{2}$, \\ Ciaran Bannan ${ }^{3}$, Carmel Waldron ${ }^{1}$, Christopher Larry Bacon ${ }^{1}$, Elisabeth Vandenberghe ${ }^{1,4}$ \\ ${ }^{1}$ Department of Haematology, St James's Hospital, Dublin, Ireland \\ ${ }^{2}$ Department of Virology, St James's Hospital, Dublin, Ireland \\ ${ }^{3}$ Department of Infectious Diseases, St James's Hospital, Dublin, Ireland \\ ${ }^{4}$ Department of Haematology, Trinity College, University of Dublin, Dublin, Ireland
}

Email address:

Jamespdillon1@gmail.com (J. Dillon)

\section{To cite this article:}

James Dillon, Elizabeth Higgins, Carmel-Ann Galligan, Majella Moran, Brendan Crowley, Ciaran Bannan, Carmel Waldron, Christopher Larry Bacon, Elisabeth Vandenberghe. Relapsed Mantle Cell Lymphoma, Allogenic Stem Cell Transplantation and COVID-19. American Journal of Internal Medicine. Vol. 9, No. 1, 2021, pp. 49-51. doi: 10.11648/j.ajim.20210901.17

Received: January 11, 2021; Accepted: January 25, 2021; Published: January 30, 2021

\begin{abstract}
The standard of care for fit patients with Mantle Cell Lymphoma (MCL) includes cytarabine containing induction treatment and consolidation with BEAM (Carmustine, Etoposide, Cytarabine and Melphalan). Chemo-refractory cases have a poorer prognosis and are candidates for allogenic stem cell transplantation (allo-SCT). Targeted therapy, such as BTK-inhibitors or BCL-mimetics can be used to bridge patients to SCT. COVID-19, a novel coronavirus has the potential to cause life threatening immune dysregulation and cytokine release syndrome (CRS) resulting in respiratory failure. Haematology patients, particularly post allogenic stem cell transplant, are high risk for developing CRS due to T-lymphopenia. PD, a 55-year-old male, with chemo-refractory MCL tested positive for COVID-19 day +45 post allo-SCT after presenting with mild gastrointestinal symptoms and remained positive for 70 days. At day +92 relapse was confirmed by CT and axillary node biopsy. Ibrutinib, a BTK inhibitor, was commenced with resolution of symptoms and a negative test within 20 days. With minimal reduction in adenopathy, Ibrutinib was stopped at day +110 , while Cytarabine and Venetoclax (BCL-mimetic) were commenced with Donor lymphocyte infusion at day +145 resulting in complete remission. Ibrutinib's therapeutic role against COVID-19 is now being investigated in clinical trials.
\end{abstract}

Keywords: Mantle Cell Lymphoma, Allogenic Stem-Cell Transplantation, COVID-19, Ibrutinib, BTK-inhibitor, Venetoclax, BCL2 Mimetic, Graft Versus Host Disease

\section{Introduction}

Ara-C (Cytarabine) containing induction treatment and high dose consolidation with BEAM (Carmustine, Etoposide, Cytarabine and Melphalan) is the standard of care for fit patients with Mantle Cell Lymphoma (MCL) resulting in survivals of $65-75 \%$ at 5 years $[1,6]$ and $64 \%$ at 10 years [3]. Patients with chemo-refractory disease have a poor prognosis, are candidates for allogenic stem cell transplantation (allo-SCT) [12] and can be bridged to SCT with either targeted therapy (BTK-inhibitor or BCL2-mimetic) or alternative chemo-immunotherapy. Chemo-refractory patients are more likely to have pleomorphic or blastoid MCL often in the context of a TP53 mutation.

COVID-19 is a novel corona virus with a variable clinical presentation from an asymptomatic to life threatening disease caused by immune dysregulation resulting in a cytokine release syndrome (CRS) with elevated inflammatory cytokine levels of IL-2, IL-6, IL-10 and TNF- $\alpha$ causing respiratory failure [7]. Haematology patients, particularly in the post allo-SCT setting are T cell-depleted and T-lymphopenia is a well-documented risk factor for COVID19-associated CRS [2]. In contrast BTK inhibitors appear to have a protective effect and clinical trials on their use in COVID-19 infected patients without B-lymphoid malignancies is currently being explored $[13,15]$. 


\section{Clinical Case}

PD is a 55-year-old male with blastoid MCL (TP53 unmutated) refractory to the Nordic protocol, who achieved a partial remission with R-DHAOx (Rituximab, Dexamethasone, Cytarabine and Oxaliplatin) and proceeded to a reduced intensity (RIC) fludarabine/melphalan/alemtuzumab matched unrelated donor (MUD) allo-SCT with ciclosporine prophylaxis. On day +45 he presented with persistent nausea and vomiting and tested positive for COVID-19 by Multiplex SARS-COV-2 RNA reverse-transcriptase real-time PCR assay. He remained well with minimal nausea, a normal inflammatory marker panel (IL-6, ferritin, CD25, CRP and Ferritin) assessed in an institutional COVID-19 clinical trial [5] and bi-weekly COVID-19 tests remained positive. On day +92 , cervical adenopathy developed and progressive MCL was confirmed on CT and axillary node biopsy. Ciclosporine was stopped and Ibrutinib $560 \mathrm{mg}$ daily was initiated resulting in resolution of nausea and a negative COVID-19 test within 20 days, but minimal improvement in MCL-associated adenopathy and no Graft versus Host $(\mathrm{GvH})$ disease. Day 100 total and CD3+ chimerism was $96 \%$ and $45 \%$ respectively. On day +110 Ibrutinib was stopped and chemotherapy with Ara-C followed by Venetoclax (a BCL2 mimetic) 400mg daily was given to clinical remission. Donor Lymphocyte infusion (DLI) at $0.3 \mathrm{x}$ $10^{7} / \mathrm{Kg} \mathrm{CD} 3+$ cells was infused on day +145 , resulting in grade $3 \mathrm{GvH}$ disease of skin and gut on day 23 (of DLI), which was controlled with Tacrolimus and Prednisolone at $2 \mathrm{mg} / \mathrm{Kg}$. He is now 269 days post allograft and in radiological complete remission (CR) from MCL with controlled GVHD and increasing donor chimerism (total 99\% and CD3+81\%).

\section{Discussion}

PD received an alemtuzumab containing allo-SCT conditioning prior to the emergence of COVID-19 in Ireland; alemtuzumab results in profound and long-lasting T-lymphocyte depletion for up to 9 months [9]. Despite this, PD had mild gastrointestinal COVID-19 symptoms, though with prolonged viral shedding for 70 days. Immunosuppressed patients (particularly post allo-SCT) excrete virus for longer than immune-competent patients [11], so the persistence of COVID-19 excretion in PD for more than 14 days was not unexpected. Alemtuzumab suppression of $\mathrm{T}$ lymphocyte function and pan-suppression of the immune system may have been paradoxically protective in preventing CRS despite the 70-day immune system exposure to COVID-19 [9]. The introduction of Ibrutinib for post-allograft MCL control, may have contributed to virus clearance within 20 days, consistent with the hypothesis that Ibrutinib is therapeutic in COVID-19 infections and is now in clinical trials [15]. Blocking BTK is hypothesised to prevent or decrease CRS by reducing the pro-inflammatory cytokines which drive acute lung injury [8]. Ibrutinib's off target inhibition of IL-2-inducible T-cell kinase (ITK) is hypothesised to enhance adaptive immunity by improving both the T-Cell function and number needed to target virally infected cell and reduce viral replication [10].

Allo-SCT results in a prolonged remission/cure in up to $40 \%$ of chemo-resistant MCL; the value of combining BCL2 mimetic therapy with graft versus lymphoma effect has resulted in a $\mathrm{CR}$ in this patient despite a post-allograft progression [4, 12]. The recent publication indicating that Chimeric Antigen Receptor T-Cell (CAR-T) therapy may provide an effective and less immunosuppressive approach for MCL is welcome [14].

\section{Conclusion}

In summary PD was Coronavirus infected from days 45 to 110 of an alemtuzumab-based RIC-MUD allo-SCT for chemo-refractory MCL causing low-grade gastrointestinal symptoms and prolonged viral shedding, which resolved within 20 days of starting Ibrutinib in keeping with its anti-COVID-19 effects. The therapeutic role of Ibrutinib against COVID-19 appears promising and is currently being investigated in clinical trials, providing a glimmer of hope in the ongoing pandemic. Chemo-refractory blastoid MCL has a poor prognosis, but PD is in remission for the first time since diagnosis following BCL2-mimetic therapy combined with DLI-induced graft versus lymphoma effect. Alemtuzumab containing allo-SCT conditioning causes prolonged T-Cell depletion increasing the risk of contracting COVID-19. Whether or not Alemtuzumab's pan-suppression of the immune system is paradoxically CRS protective, CAR-T may now offer an effective, less immunosuppressive treatment for MCL, which is particularly relevant in the current COVID-19 pandemic.

\section{Conflicts of Interest}

The authors declare that they have no competing interests.

\section{References}

[1] Delarue, R., Haioun, C., Ribrag, V., Brice, P., Delmer, A., \& Tilly, H. et al. (2013). CHOP and DHAP plus rituximab followed by autologous stem cell transplantation in mantle cell lymphoma: a phase 2 study from the Groupe d'Etude des Lymphomes de l'Adulte. Blood, 121 (1), 48-53. doi: 10.1182/blood-2011-09-370320.

[2] El-Sharkawi, D., \& Iyengar, S. (2020). Haematological cancers and the risk of severe COVID-19: Exploration and critical evaluation of the evidence to date. British Journal Of Haematology, 190 (3), 336-345. doi: 10.1111/bjh.16956.

[3] Eskelund, C., Kolstad, A., Jerkeman, M., Räty, R., Laurell, A., $\&$ Eloranta, S. et al. (2016). 15-year follow-up of the Second Nordic Mantle Cell Lymphoma trial (MCL2): prolonged remissions without survival plateau. British Journal of Haematology, 175 (3), 410-418. doi: 10.1111/bjh.14241.

[4] Eyre, T., Walter, H., Iyengar, S., Follows, G., Cross, M., \& Fox, C. et al. (2018). Efficacy of venetoclax monotherapy in patients with relapsed, refractory mantle cell lymphoma after Bruton tyrosine kinase inhibitor therapy. Haematologica, 104 (2), e68-e71. doi: 10.3324/haematol.2018.198812. 
[5] Fogarty, H., Townsend, L., Ni Cheallaigh, C., Bergin, C., Martin-Loeches, I., \& Browne, P. et al. (2020). COVID19 coagulopathy in Caucasian patients. British Journal Of Haematology, 189 (6), 1044-1049. doi: 10.1111/bjh.16749.

[6] Hermine, O., Hoster, E., Walewski, J., Bosly, A., Stilgenbauer, S., \& Thieblemont, C. et al. (2016). Addition of high-dose cytarabine to immunochemotherapy before autologous stem-cell transplantation in patients aged 65 years or younger with mantle cell lymphoma (MCL Younger): a randomised, open-label, phase 3 trial of the European Mantle Cell Lymphoma Network. The Lancet, 388 (10044), 565-575. doi: 10.1016/s0140-6736(16)00739-x.

[7] Kakodkar, P., Kaka, N., \& Baig, M. (2020). A Comprehensive Literature Review on the Clinical Presentation, and Management of the Pandemic Coronavirus Disease 2019 (COVID-19). Cureus. doi: 10.7759/cureus. 7560.

[8] Lin, A., Cuttica, M., Ison, M., \& Gordon, L. (2020). Ibrutinib for chronic lymphocytic leukemia in the setting of respiratory failure from severe COVID-19 infection: Case report and literature review. Ejhaem, 1 (2), 596-600. doi: 10.1002/jha2.98.

[9] Matías-Guiu, J., Montero-Escribano, P., Pytel, V., Porta-Etessam, J., \& Matias-Guiu, J. (2020). Potential COVID-19 infection in patients with severe multiple sclerosis treated with alemtuzumab. Multiple Sclerosis And Related Disorders, 44, 102297. doi: 10.1016/j.msard.2020.102297.
[10] McGee, M., August, A., \& Huang, W. (2020). BTK/ITK dual inhibitors: Modulating immunopathology and lymphopenia for COVID-19 therapy. Journal Of Leukocyte Biology. doi: 10.1002/jlb.5covr0620-306r.

[11] Ogimi, C., Greninger, A., Waghmare, A., Kuypers, J., Shean, R., \& Xie, H. et al. (2017). Prolonged Shedding of Human Coronavirus in Hematopoietic Cell Transplant Recipients: Risk Factors and Viral Genome Evolution. The Journal Of Infectious Diseases, 216 (2), 203-209. doi: 10.1093/infdis/jix264.

[12] Roué, G., \& Sola, B. (2020). Management of Drug Resistance in Mantle Cell Lymphoma. Cancers, 12 (6), 1565. doi: $10.3390 /$ cancers 12061565 .

[13] Treon, S., Castillo, J., Skarbnik, A., Soumerai, J., Ghobrial, I., \& Guerrera, M. et al. (2020). The BTK inhibitor ibrutinib may protect against pulmonary injury in COVID-19-infected patients. Blood, 135 (21), 1912-1915. doi: 10.1182/blood.2020006288.

[14] Voelker, R. (2020). CAR-T Therapy Is Approved for Mantle Cell Lymphoma. JAMA, 324 (9), 832. doi: 10.1001/jama.2020.15456.

[15] Woyach, J. (2020). Ibrutinib for the Treatment of COVID-19 in Patients Requiring Hospitalization - Full Text View ClinicalTrials.gov. Retrieved 29 November 2020, from https://clinicaltrials.gov/ct2/show/NCT04439006. 